\title{
The rediscovery of slowness: exploring the timing of cognition
}

\author{
Kringelbach, Morten L. ${ }^{1,2}$, McIntosh, Anthony R. ${ }^{3}$, Ritter, Petra ${ }^{4,5}$ Jirsa, Viktor K. ${ }^{6}$, Deco, Gustavo ${ }^{7,8}$
}

1 Department of Psychiatry, University of Oxford, Oxford OX3 7JX, UK

2 Center of Functionally Integrative Neuroscience (CFIN), Aarhus University, DK

3 Rotman Research Institute of Baycrest Center, University of Toronto, Toronto, M6A 2E1, Canada

4 Max-Planck Institute for Cognitive and Brain Sciences, Leipzig, Germany

5 Department of Neurology, Charité, Charitéplatz 1, 10117 Berlin

6 Institut de Neurosciences des Systèmes UMR INSERM 1106, Aix-Marseille Université, Faculté de Médecine, 27, Boulevard Jean Moulin, 13005 Marseille, France

7 Center for Brain and Cognition, Computational Neuroscience Group, Department of Information and Communication Technologies, Universitat Pompeu Fabra, Roc Boronat 138, Barcelona, 08018, Spain

8 Institució Catalana de la Recerca i Estudis Avançats (ICREA), Universitat Pompeu Fabra, Passeig Lluís Companys 23, Barcelona, 08010, Spain

Corresponding author:

Gustavo Deco, Universitat Pompeu Fabra, C/ Roc Boronat, 138, 08018 Barcelona, Spain. gustavo.deco@upf.edu, Tel: 0034-935422977, Fax: 0034-935422517

Conflict of interest: the authors declare to have no conflict of interest.

Running Title: Rediscovery of slowness

Keywords: Resting-state activity; Whole-brain modelling; Dynamical systems

\begin{abstract}
Slowness of thought is not necessarily a handicap but could be a signature of optimal brain function. Emerging evidence shows that neuroanatomical and dynamical constraints of the human brain shape its functionality in optimal ways, characterized by slowness during task-based cognition in the context of spontaneous resting-state activity. This activity can be described mechanistically by whole-brain computational modelling, related directly with optimality in the context of theories arguing for metastability in the brain. We discuss the role for optimal processing of information in the context of cognitive, taskrelated activity and propose that combining multi-modal neuroimaging and explicit whole-brain models focused on the timing of functional dynamics can help uncover fundamental rules of brain function in health and disease.
\end{abstract}




\section{Rediscovering slowness}

In his thoughtful and beautifully written book "Die Entdeckung der Langsamkeit" (transl. "The Discovery of Slowness") [1], the German author Sten Nadolny meditates over the ultimate reasons for the success and failure of the English polar researcher John Franklin (1786-1847). Franklin was much celebrated during the Victorian era but perished on his third expedition to discover the mythical Northwest Passage, supposed to link the Atlantic and the Pacific Oceans above the northern coast of the American continent. In the novel, Nadolny suggests that Franklin's fatal attraction to the Arctic was linked to his desire to find an environment suited to his slowness of thought, which among other things manifested itself in his inability to speak fluently, memorising instead 'entire fleets of words and batteries of response'. Nadolny makes the compelling argument that it is the slowness of cognition, which is an essential component of optimality of thought.

This literary view of slowness resonates well with dual process theories $[2,3]$, and in particular the work of Daniel Kahneman and Amos Tversky, who collected intriguing behavioural evidence that the human mind consists of two competing fast and slow systems [4, 5]. System 1 is fast, instinctive and emotional, whereas System 2 is much slower, more deliberative, and more logical. System 2 has limited access to System 1, which is the main source of impressions, intuitions, intentions and emotions, but it will still consciously elaborate on these and eventually turn them into beliefs and voluntary actions. A firm neurobiological foundation of these systems has not yet been established but there is mounting evidence that these systems are the result of the dynamics of interacting networks of brain regions over time rather than the contribution of any single region [6].

The separation of timescales of neural functioning has also been put forward in modern computational theories of information processing in human behaviour [7-9] and brain disorders such as epilepsy [10]. Inspired by the theory of nonlinear dynamical systems, timescale separation offers, first, a means to separate two sets of variables functionally and, second, to build hierarchies of processes constituting cognitive architectures. Intuitive examples include, for instance, handwriting comprising dynamic behaviour on fast and slow timescales, from creating the individual letters forming words, sentences and paragraphs [11].

In this Review, we expand on these ideas to show how slowness is not solely a literary construct but could be a fundamental principle of brain function, emerging from underlying brain dynamics as demonstrated by advanced whole-brain computational modelling. We briefly review the rise of resting-state studies that focus on intrinsic brain dynamics in basic and clinical neuroscience, but show how the analysis of time in the brain has become increasingly important. In particular, recent findings [12,13] have radically shifted the emphasis from static spatial correlation analysis to time-dependent analysis of the fluctuations and dynamics of functional processing. We then review the role of whole-brain computational modelling for the growing understanding of the dynamics of brain function, and emphasize how these models can lead to a deeper understanding of the spatiotemporal dynamics. We show how modelling has been used to shed new light on the metastable properties of the human brain, i.e. states falling outside the natural equilibrium state of the system but persisting for an extended period of time. This has demonstrated that the healthy human brain is maximally metastable, which leads to a natural slowness of task-related cognition. Finally, we 
discuss how these new findings of slowness in an era of relentless speed may come to have profound influence on our understanding of brain function in health and disease.

\section{Characterising functional and structural brain connectivity}

Over the last decade, there has been an explosion of empirical and theoretical interest in the spontaneous or intrinsic activity of the brain [14]. Initially, neuroimaging experiments assumed that this spontaneous activity was mainly noise and could be used simply as a control for discovering task-related activity $[15,16]$. Yet, careful experiments using a wide variety of neuroimaging techniques have found that spontaneous activity is highly structured in time and space [17-19]. Neuroimaging experiments uncovered large distributed networks of brain areas that are highly correlated, and were named resting state networks (RSN) [20,21] - although this is really a misnomer given that their very existence demonstrates that resting brains never rest $[22,23]$. These RSN can be quantified in terms of functional connectivity (FC), which can be expressed as a matrix containing the correlations across a long time series between all pairs of brain areas (at the voxel level or more typically at the level of a given standard brain parcellation (e.g. [24, 25]). Typically this FC matrix quantifies the average spatial structure of the RSN (see Figure 1).

Still, the question remains of how these correlations emerge in the first place. One simple idea is that they could be tied to the underlying structure of the anatomical connections linking them; in many ways akin to the ideas of the medieval philosopher Thomas Aquinas who, in the spirit of Aristotle, wrote "Quidquid recipitur ad modum recipientis recipitur", i.e. the container (or recipient) shapes the content. In the case of the brain, the underlying anatomy has been proposed to shape the functional activity $[26,27]$. The argument goes that even if the local neuronal dynamics in each single node (e.g. a voxel) is mainly noise (as evidenced by neurophysiological recordings [28-30]), these voxels are linked by anatomical myelinated fibres connecting them synaptically, and thus their correlations could be driven and shaped by those links.

This attractive idea would be testable if it were possible to extract the anatomical structural skeleton, i.e. the structural connectivity (SC) that can be expressed in terms of a structural connectivity matrix. This can be mapped in vivo in humans on the scale of millimetres using diffusion weighted/tensor imaging (DWI/DTI) which can measure the white-matter fiber tracts constrained by the diffusion of water molecules [31, 32] and where the connectivity between brain regions can be reconstructed by probabilistic tractography [33, 34] (Figure 1A).

Over time, evidence for this hypothesis has accumulated from a number of sources, including seminal research in primates, showing that the average FC is significantly correlated with the SC during rest ([26]; Figure 1C). Still, this group and others only found low, albeit significant, structure-function correlations, suggesting that there are other factors shaping brain activity. Perhaps the most important clue to other potential influences comes from the fact that the typical FC matrix calculation ignores the importance of time. We and other researchers have argued that dynamics are indeed crucial for understanding how the FC is shaped, i.e. in addition to the SC others factors such as time delays and the current global working point of the brain [13]. In other words, careful dynamical analysis is likely to improve our understanding of the underlying spontaneous brain activity. 


\section{Uncovering the spatiotemporal dynamics of brain activity}

Studies have demonstrated that the correlations among brain regions, both within and between networks, evolve over time [35-37]. Important insights was recently made by the observations of fluctuations of the FC when calculated over relatively short sliding windows [12]. Further progress was made quantifying the spatiotemporal structure of these fluctuations by introducing a careful dynamic analysis characterising the FC dynamics (FCD) and explaining its nature based on large-scale network models [13]. The method for calculating the FCD is summarised in Figure 2.

When applied to empirical data, the FCD matrices show a characteristic checkerboard-like structure signalling when there is switching between different brain states or activity patterns (see Figure $2 \mathrm{C}$ ). In other words, the analysis can show when a brain network state is reoccurring intermittently over time, across scanning sessions and even across participants. These results suggest that spatial patterns are formed, dissolved, and reformed over time, so that periods of resting activity can be subdivided into subsets or 'communities' of brain regions that strongly interact over a period of time.

Research has demonstrated that the global phase synchrony of the timeseries of brain activity evolves on a characteristic ultra-slow $(<0.01 \mathrm{~Hz})$ timescale and that its temporal variations reflect the transient formation and dissolution of multiple communities of synchronized brain regions [38]. The level of variability of the global phase synchronisation can be expressed by the standard deviation of the so-called Kuramoto order parameter, defining the metastability of a given system. Empirical measurements have confirmed that the brain is metastable [39].

Importantly, such time-varying functional connectivity has been reported both in awake humans and anesthetized macaques $[36,40]$, thus the temporal variability of functional interactions is not likely to be produced by transitions between different mental states, but instead may be an emergent property of complex brain networks. These results suggest that the interplay between space and time in the brain is crucial to unravel the mechanistic origins of spontaneous activity.

\section{Whole-Brain Computational Modelling}

One powerful way to investigate and model the non-stationary dynamics of whole-brain activity is to use whole-brain computational modelling (see Box 1), which takes into account the interplay and mutual entrainment of local dynamics using the underlying structural connections and results in global whole-braindynamics (see Figure 3A). Indeed, whole-brain models have been shown to be able to explain the spatial aspects of the resting FC by fitting the spatial correlation as extracted by the grand average FC [23, 41]. Research has shown that the fitting of the grand average FC overemphasizes the influence of the SC and the resulting models are incapable of showing the non-stationary switching dynamics of the FC [13]. This difference is qualitative and cannot be simply addressed by quantitative parameter tuning. Following this insight the fitting of FC at one timescale only was abandoned and instead it was shown that a slight increase in nonlinearity of the node models suffices to create the non-stationary FCD as observed empirically. In fact, 
it appears that the slow switching of FC dynamics is the normal behaviour of a nonlinear large-scale brain network and occurs naturally without any major parameter tuning.

So the question arises what is the purpose, if any, of the slow FCD? Mechanistically, the most detailed models describe local spontaneous dynamics as attractors by means of explicit simulations of spiking neurons and their detailed synaptic connections including the dynamics of specific neurotransmitter receptor types [42]. Yet, the emergent collective macroscopic behaviour of brain models has been shown to be only weakly dependent on the details of individual neuron behaviour [43].

This agrees with fundamental results from statistical physics, which have shown that macroscopic physical systems obey laws that can be independent of their mesoscopic constituents [44]. In turn, this allows whole-brain modelling to use reduced neural mass models, of which there are two main types: 1) oscillatory models [45-50] and 2) asynchronous models [51-53]. These models can be unified by using a neural mass local model, such as the normal form of a Hopf bifurcation, to describe the transition from noise to oscillations, which has been shown to exhibit both richness and generality in describing the temporal dynamics of EEG at the local node level [54, 55].

\section{The Resting Brain is Maximally Metastable and Slow}

The evidence from a wide range of experiments suggests that the brain is metastable [39] (see Box 2). Further empirical evidence has recently come from whole-brain computational models with oscillatory local nodes using the Kuramoto model $[50,56]$. These simulations fitting the model to MEG data recorded over milliseconds importantly show that the brain is not only metastable, but maximally metastable, which implies the possibility for the widest exploration of the dynamical repertoire of the network. The Kuramoto model was implemented within the AAL parcellation of the structural connectivity of the human brain and used only two parameters, namely the global coupling strength and the mean delay between brain regions. As shown in Figure 3B, the model fits the data optimally within a limited combination of values of the two parameters as shown in the plots of 1) goodness of fit to empirical FC data, 2) mean and 3) standard deviation of synchrony degree and 4) standard deviation of the $8-30 \mathrm{~Hz}$ envelopes. Perhaps most importantly, at this particular working point of the whole-brain model, metastability is also maximal, which potentially has deep significance for our understanding of fundamental brain function.

It is important to realise that this type of discovery can only be made through a combination of integration and fitting of multimodal neuroimaging data with computational models [42]. Conventional correlational analyses are able to demonstrate that the brain is metastable, but crucially not whether it is maximally metastable - this is only possible with whole-brain computational modelling.

One of the important consequences of the brain being maximally metastable is that this implies a characteristic slowness of spontaneous dynamics (i.e. the switching transitions between brain states) upon approaching a bifurcation or state transition; a phenomenon called critical slowing down. While predicted by theory, only recently has this been demonstrated experimentally in an elegant whole-cell patch-clamp recordings from pyramidal neurons and fast-spiking interneurons in rats [57]. Switching dynamics and its critical co-occurring phenomena have been previously observed in human behaviour [58] and human brain 
recordings $[59,60]$. It is the propagation of switching behaviour across brain scales, i.e. microscopic to macroscopic, that is relevant for cognition [61].

Whole-brain computational models can also be used to study the temporal evolution of resting state activity using the Kuramoto order parameter to measure the global level of synchronisation of the oscillating signals between brain regions [38]. Figure 3C shows an example of this temporal evolution of the global level of synchronization in the human brain. Notably, the power spectrum of this evolves on an ultra-slow $(<0.01 \mathrm{~Hz})$ timescale (Figure 3D). Similarly, whole-brain computational models have demonstrated a critical slowing down on the edge of a criticality (Figure 3E) [51]. These findings imply optimality in the sense of maximally and wide exploration of the dynamical repertoire of the network, which is a fundamental property for optimal task processing.

\section{Beyond Resting: The Brain is Slow under Stimuli/Task Processing}

One must question the importance of slowness in the resting brain when it could be argued that cognition is more about engaging with tasks and stimuli than what is done at rest [16]. From the very start, neuroimaging experiments have - albeit mostly involuntarily - explored the link between spontaneous and task-related activity by pervasive use of subtraction paradigms. Yet, it is only with the rise of resting state paradigms that a better understanding has arisen of the link between them $[23,62]$.

Neuroimaging experiments have started to reveal some of the key differences between spontaneous and task-related activity in more well-controlled ways and in particular to quantify the speed of processing. One recent experiment assessed brain activity patterns in response to a natural slow, temporal sequence of images (movie), which was scrambled over time (scrambled movie) or space (noise); thus preserving the first-order statistics across stimuli [63] (Figure 4A). Strong and widespread cortical activity was found in all conditions, but selectively the Lempel-Ziv complexity of these activity patterns was found to have the highest value for the movie and minimal for the noise condition. Thus, while the stimuli were carefully matched, meaningful integration and segregation of externally driven information over time clearly rely on slow processing of natural, temporally structured information.

Similarly, studies in other animals have established links between spontaneous activity and externally driven information. For example, a recording study in awake ferrets found a strong similarity between stimulus-evoked and spontaneous neural activities to responses evoked by natural scenes [64] (Figure 4B). They found improvements to this activity during development, which demonstrates how the brain clearly adapts to the slowness of natural stimuli at the neural level.

Yet, these are correlational results, and whole-brain computational modelling can provide new evidence for what happens under stimuli/task processing, where the evoked brain activity is designed for optimal processing and where encoding external information provided at an optimal "slow" range of latencies (i.e. at the timing of cognition).

We hypothesize that it should be possible to measure the binding capability of the human brain as a function of the latency of the external stimulation. Binding is defined as the amount of information of the input signal which is encoded in the network across time, i.e. the entropy of all nodes across time [65]. The 
link to stimulus-evoked activity can be explored by simulating natural, external stimulation using the wellknown independent stochastic (Ornstein-Uhlenbeck) process with equal variance but different latencies [66]. Subsequently, this would allow for measurement of the binding of information over time by the brain activity using a sliding window approach (see Figure 4C) [65]. Existing evidence of ultra-slow activity [38] suggests that a clear maximum will emerge around 20 seconds, which means that around this relatively slow time point, the brain is able to optimally process external inputs. In other words, in order to process optimally external time signals, their latencies should be on the order of the underlying latency of the timing of the resting brain, i.e. of the switching between resting brain states. At that point, the external signal "resonates" with the underlying latency of the whole brain dynamics and therefore the processing is optimal.

As shown earlier, this slowness in processing, or the latency of the metastability, is the result of the resonance of the latency of the external inputs with the critical slowing down or intrinsic slowness of the switching between the different brain states (at rest). Thus, importantly, this finding is unlikely to be an artefact of e.g. low pass filtering of the BOLD signal.

\section{Concluding Remarks and Perspectives}

Slowness is not obviously a desirable quality in a ruthless natural world built primarily on survival; yet the relative evolutionary success of humans is evidence that a certain slowness of cognition combined with fast reactions can be highly adaptive. The flexibility of human cognition for goal-directed behaviour relies on our ability to build coherent, distributed dynamical states by segregating and integrating information on multiple timescales [65].

As an example, think about how helpless human infants are during infancy and childhood, and how caregiving behaviour must be key to the survival of the human species [67, 68]. Care-giving behaviour depends partly on very fast processing of infant cues, exemplified by the fact that infant sounds elicit significant activity already after only 50ms in the brainstem [69] and infant facial features elicit activity in the orbitofrontal cortex within around 130ms [70]. Yet, once this attentional, orienting response has taken place, it is replaced with careful, slow caregiving behaviour that can address the specific needs of the infant. This slower, deliberate cognition has been shown to rely on much later brain activity in distributed networks which include the very same regions of the orbitofrontal cortex recruited earlier for the fast response [71]. This is potentially important for the future well-being of the infant given how slow, maladaptive parental cognitions such as rumination and worry can lead to significant disturbances in the parent-infant relationship [72]. More generally, the dual process of fast, attention-orienting activity followed and modulated by slower, conscious cognitive processes has been shown to be a recurrent theme across the sensory modalities and highly dependent on intact reward processing [73-75].

What has been missing is a mechanistic understanding of how the human brain achieves the right balance between the slow and fast processes across temporal hierarchies in cognitive architectures [7]. Here, we have shown the emerging evidence that during task processing, the maximally metastable brain contains intrinsic brain activity that is designed for optimal processing, and can encode external information at an optimal "slow" range of latencies. 
This new insight into striking the optimal balance between fast and slow cognition may also be important for understanding the progression in brains from healthy to diseased. As such, it adds to current ideas of using connectomics in neuropsychiatry to discover new biomarkers and potential treatments [76] by using whole-brain computational models for understanding fundamental principles of brain function, especially to further understand the importance of time [53]. As a result, the field is now beginning to shift from using static grand averages (based on connectomics) to characterizing the time-dependent dynamics of whole-brain networks. It would be of considerable interest to test whether slowness could be an essential biomarker of a healthy brain (see Outstanding Questions Box).

Overall, the rediscovery of time in cognitive neuroscience and connectomics is leading to new insights into the fundamental principles of brain function. Not only may this help to better understand the human brain's precarious balancing act of processing and predicting stimuli on different timescales, whether slow or fast - but it may also lead to a new appreciation of how slowness can help us deal with what may feel like an ever accelerating pace of life. Nadolny suggests that Franklin may have sought the Arctic to match the speed of his thought processes, but ultimately the most important factor is the balance between fast and slow, between adequately processing the past to predict not just the now but the future. As observed by the Polish poet Wislawa Szymborska "when I pronounce the word Future, the first syllable already belongs to the past." Yet, by learning to optimally balance fast and slow processes in the maximally metastable brain, we may be able to extend the now into the long now. This balancing act could potentially be extended to a Zen-like perspective of observing infinite gratitude for the past, infinite service to the present, and infinite responsibility for the future. Although such balanced states are rare, science reminds us that a potential route could be through the built-in slowness of the architecture of our brains.

\section{Acknowledgements}

MLK is supported by the ERC Consolidator Grant: CAREGIVING (n. 615539). GD is supported by the ERC Advanced Grant: DYSTRUCTURE (n. 295129) and by the Spanish Research Project PSI2013-42091P. In addition, the authors acknowledge the support of the German Ministry of Education and Research (Bernstein Focus State Dependencies of Learning 01GQ0971-5 to PR, the James S. McDonnell Foundation (Brain Network Recovery Group JSMF22002082) to ARM, GD, PR and VJ, the Max-Planck Society (Minerva Program) to PR, and funding from the European Union Seventh Framework Programme (FP7-ICT Human Brain Project (grant no. 60402)) to GD, VJ. 


\section{References}

1 Nadolny, S. (1983) Die Entdeckung der Langsamkeit (transl. The Discovery of Slowness). Piper

2 Stanovich, K.E. and West, R.F. (2000) Individual differences in reasoning: implications for the rationality debate? The Behavioral and brain sciences 23, 645-665; discussion 665-726

3 Posner, M.I. and Snyder, C.R.R. (1975) Attention and cognitive control. In Information processing and cognition: The Loyola Symposium (Solso, R.L., ed), Wiley

4 Tversky, A. and Kahneman, D. (1974) Judgment under Uncertainty: Heuristics and Biases. Science 185, 1124-1131

5 Kahneman, D. (2011) Thinking, fast and slow. Farrar, Straus \& Giroux

6 Varela, F., et al. (2001) The brainweb: phase synchronization and large-scale integration. Nature reviews. Neuroscience 2, 229-239

7 Huys, R., et al. (2014) Functional architectures and structured flows on manifolds: a dynamical framework for motor behavior. Psychol Rev 121, 302-336

8 Perdikis, D., et al. (2011) Time scale hierarchies in the functional organization of complex behaviors. PLoS computational biology 7, e1002198

9 Woodman, M., et al. (2011) Building neurocognitive networks with a distributed functional architecture. Advances in experimental medicine and biology 718, 101-109

10 Jirsa, V.K., et al. (2014) On the nature of seizure dynamics. Brain : a journal of neurology 137, 2210-2230

11 Perdikis, D., et al. (2011) Complex processes from dynamical architectures with time-scale hierarchy. PloS one 6, e16589

12 Allen, E.A., et al. (2014) Tracking whole-brain connectivity dynamics in the resting state. Cerebral cortex 24, 663676

13 Hansen, E.C., et al. (2015) Functional connectivity dynamics: modeling the switching behavior of the resting state. NeuroImage 105, 525-535

14 Biswal, B.B., et al. (2010) Toward discovery science of human brain function. Proceedings of the National Academy of Sciences of the United States of America 107, 4734-4739

15 Raichle, M.E. and Snyder, A.Z. (2007) A default mode of brain function: a brief history of an evolving idea. NeuroImage 37, 1083-1090; discussion 1097-1089

16 Morcom, A.M. and Fletcher, P.C. (2007) Does the brain have a baseline? Why we should be resisting a rest. NeuroImage 37, 1073-1082

17 Raichle, M.E., et al. (2001) A default mode of brain function. Proceedings of the National Academy of Sciences of the United States of America 98, 676-682

18 Beckmann, C.F., et al. (2005) Investigations into resting-state connectivity using independent component analysis. Philos. Trans. R. Soc. Lond. B Biol. Sci. 360, 1001-1013

19 Fransson, P. (2005) Spontaneous low-frequency BOLD signal fluctuations: an fMRI investigation of the resting-state default mode of brain function hypothesis. Human brain mapping 26, 15-29

20 Biswal, B., et al. (1995) Functional connectivity in the motor cortex of resting human brain using echo-planar MRI. Magnetic resonance in medicine : official journal of the Society of Magnetic Resonance in Medicine / Society of Magnetic Resonance in Medicine 34, 537-541

21 Fox, M.D., et al. (2005) The human brain is intrinsically organized into dynamic, anticorrelated functional networks. Proceedings of the National Academy of Sciences of the United States of America 102, 9673-9678

22 Bullmore, E. and Sporns, O. (2009) Complex brain networks: Graph theoretical analysis of structural and functional systems. Nature Reviews Neuroscience 10, 186-198

23 Deco, G., et al. (2013) Resting brains never rest: computational insights into potential cognitive architectures. Trends in neurosciences $36,268-274$

24 Tzourio-Mazoyer, N., et al. (2002) Automated anatomical labeling of activations in SPM using a macroscopic anatomical parcellation of the MNI MRI single-subject brain. NeuroImage 15, 273-289

25 Desikan, R.S., et al. (2006) An automated labeling system for subdividing the human cerebral cortex on MRI scans into gyral based regions of interest. NeuroImage 31, 968-980

26 Vincent, J.L., et al. (2007) Intrinsic functional architecture in the anaesthetized monkey brain. Nature 447, 83-86

27 Moeller, S., et al. (2009) Functional connectivity of the macaque brain across stimulus and arousal states. The Journal of neuroscience : the official journal of the Society for Neuroscience 29, 5897-5909

28 Shadlen, M.N. and Newsome, W.T. (1998) The variable discharge of cortical neurons: implications for connectivity, computation, and information coding. The Journal of neuroscience : the official journal of the Society for Neuroscience $18,3870-3896$

29 Softky, W.R. and Koch, C. (1993) The highly irregular firing of cortical cells is inconsistent with temporal integration of random EPSPs. The Journal of neuroscience : the official journal of the Society for Neuroscience 13, 334-350

30 Churchland, M.M., et al. (2010) Stimulus onset quenches neural variability: a widespread cortical phenomenon. Nature neuroscience 13, 369-378 
31 Basser, P.J. and Pierpaoli, C. (1996) Microstructural and physiological features of tissues elucidated by quantitativediffusion-tensor MRI. Journal of magnetic resonance. Series B 111, 209-219

32 Beaulieu, C. (2002) The basis of anisotropic water diffusion in the nervous system - a technical review. NMR Biomed 15, 435-455

33 Johansen-Berg, H. and Rushworth, M.F. (2009) Using diffusion imaging to study human connectional anatomy. Annual review of neuroscience 32, 75-94

34 Hagmann, P., et al. (2010) MR connectomics: Principles and challenges. J Neurosci Methods 194, 34-45

35 Chang, C. and Glover, G.H. (2010) Time-frequency dynamics of resting-state brain connectivity measured with fMRI. Neurolmage 50, 81-98

36 Hutchison, R.M., et al. (2013) Resting-state networks show dynamic functional connectivity in awake humans and anesthetized macaques. Human brain mapping 34, 2154-2177

37 Kiviniemi, V., et al. (2011) A sliding time-window ICA reveals spatial variability of the default mode network in time. Brain connectivity 1, 339-347

38 Ponce-Alvarez, A., et al. (2015) Resting-state temporal synchronization networks emerge from connectivity topology and heterogeneity. PLoS computational biology 11, e1004100

39 Tognoli, E. and Kelso, J.A. (2014) The metastable brain. Neuron 81, 35-48

40 Barttfeld, P., et al. (2015) Signature of consciousness in the dynamics of resting-state brain activity. Proceedings of the National Academy of Sciences of the United States of America 112, 887-892

41 Deco, G., et al. (2011) Emerging concepts for the dynamical organization of resting-state activity in the brain. Nature reviews. Neuroscience 12, 43-56

42 Deco, G. and Jirsa, V.K. (2012) Ongoing cortical activity at rest: criticality, multistability, and ghost attractors. The Journal of neuroscience : the official journal of the Society for Neuroscience 32, 3366-3375

43 Breakspear, M. and Jirsa, V. (2007) Neuronal Dynamics and Brain Connectivity. In Handbook of brain connectivity (Jirsa, V.K. and McIntosh, A.R., eds), pp. 3-64, Springer

44 Haken, H. (1975) Cooperative phenomena in systems far from thermal equilibrium and in nonphysical systems. Reviews of Modern Physics 47, 67-121

45 Cabral, J., et al. (2014) Exploring the network dynamics underlying brain activity during rest. Prog Neurobiol 114 , $102-131$

46 Deco, G., et al. (2009) Key role of coupling, delay, and noise in resting brain fluctuations. Proceedings of the National Academy of Sciences of the United States of America 106, 10302-10307

47 Ghosh, A., et al. (2008) Cortical network dynamics with time delays reveals functional connectivity in the resting brain. Cogn Neurodyn 2, 115-120

48 Ghosh, A., et al. (2008) Noise during rest enables the exploration of the brain's dynamic repertoire. PLoS computational biology 4, e1000196

49 Cabral, J., et al. (2011) Role of local network oscillations in resting-state functional connectivity. NeuroImage 57, 130-139

50 Cabral, J., et al. (2014) Exploring mechanisms of spontaneous functional connectivity in MEG: How delayed network interactions lead to structured amplitude envelopes of band-pass filtered oscillations. Neuroimage 90, 423-435

51 Deco, G., et al. (2013) Resting-state functional connectivity emerges from structurally and dynamically shaped slow linear fluctuations. The Journal of neuroscience : the official journal of the Society for Neuroscience 33, 11239-11252

52 Honey, C.J., et al. (2007) Network structure of cerebral cortex shapes functional connectivity on multiple time scales. Proceedings of the National Academy of Sciences of the United States of America 104, 10240-10245

53 Deco, G. and Kringelbach, M.L. (2014) Great Expectations: Using Whole-Brain Computational Connectomics for Understanding Neuropsychiatric Disorders. Neuron 84, 892-905

54 Freyer, F., et al. (2011) Biophysical mechanisms of multistability in resting-state cortical rhythms. The Journal of neuroscience : the official journal of the Society for Neuroscience 31, 6353-6361

55 Freyer, F., et al. (2012) A canonical model of multistability and scale-invariance in biological systems. PLoS Comput Biol 8, e1002634

56 Kuramoto, Y. (1984) Chemical Oscillations, Waves, and Turbulence. Springer-Verlag, Berlin

57 Meisel, C., et al. (2015) Critical slowing down governs the transition to neuron spiking. PLoS computational biology 11 , e1004097

58 Schoner, G. and Kelso, J.A. (1988) Dynamic pattern generation in behavioral and neural systems. Science 239, $1513-1520$

59 Jirsa, V.K., et al. (1998) Connecting cortical and behavioral dynamics: bimanual coordination. Neural computation $10,2019-2045$

60 Fuchs, A., et al. (1992) Phase transitions in the human brain: spatial mode dynamics. International Journal of Bifurcation and Chaos 2, 917-939

61 Ritter, P., et al. (2015) State-dependencies of learning across brain scales. Frontiers in computational neuroscience 9,1

62 Ritter, P., et al. (2015) Editorial: State-dependent brain computation. Front. Comput. Neurosci. 9, 77

63 Boly, M., et al. (2015) Stimulus set meaningfulness and neurophysiological differentiation: a functional magnetic resonance imaging study. PloS one 10 , e 0125337 
64 Berkes, P., et al. (2011) Spontaneous cortical activity reveals hallmarks of an optimal internal model of the environment. Science 331, 83-87

65 Deco, G., et al. (2015) Rethinking segregation and integration: contributions of whole-brain modelling Nat Rev Neurosci 16, 430-439

66 Uhlenbeck, G.E. and Ornstein, L.S. (1930) On the theory of Brownian Motion. Phys. Rev. 36, 823-841

67 Parsons, C.E., et al. (2010) The functional neuroanatomy of the evolving parent-infant relationship. Progress in Neurobiology $91,220-241$

68 Bornstein, M.H., et al. (2012) Emotional Relationships in Mothers and Infants: Culture-Common and CommunitySpecific Characteristics of Dyads from Rural and Metropolitan Settings in Argentina, Italy, and the United States. $J$ Cross Cult Psychol 43, 171-197

69 Parsons, C.E., et al. (2014) Ready for action: A role for the brainstem in responding to infant vocalizations. Social Cognitive and Affective Neuroscience 9, 977-984

70 Kringelbach, M.L., et al. (2008) A specific and rapid neural signature for parental instinct. PLoS ONE 3 , e1664. doi:1610.1371/journal.pone.0001664

71 Parsons, C.E., et al. (2013) Understanding the human parental brain: a critical role of the orbitofrontal cortex. Soc Neurosci 8, 525-543

72 Stein, A., et al. (2012) Maternal cognitions and mother-infant interaction in postnatal depression and generalized anxiety disorder. Journal of abnormal psychology 121, 795-809

73 Bar, M., et al. (2006) Top-down facilitation of visual recognition. Proceedings of the National Academy of Sciences of the United States of America 103, 449-454

74 Kringelbach, M.L. (2005) The orbitofrontal cortex: linking reward to hedonic experience. Nature Reviews Neuroscience 6, 691-702

75 Berridge, K.C. and Kringelbach, M.L. (2015) Pleasure systems in the brain. Neuron 86, 646-664

76 Rubinov, M. and Bullmore, E. (2013) Fledgling pathoconnectomics of psychiatric disorders. Trends in cognitive sciences $17,641-647$

77 Ritter, P., et al. (2013) The virtual brain integrates computational modeling and multimodal neuroimaging. Brain connectivity 3, 121-145

78 Kringelbach, M.L., et al. (2011) Balancing the brain: resting state networks and deep brain stimulation. Frontiers Integrative Neuroscience 5, 8 


\section{Figures and boxes}

A

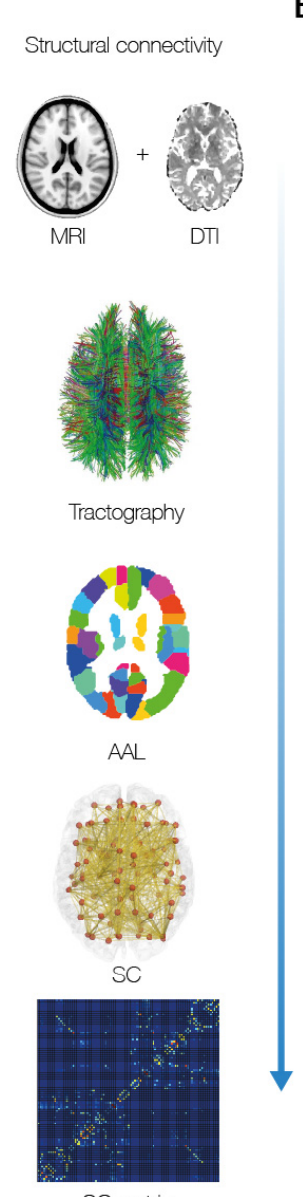

B

C

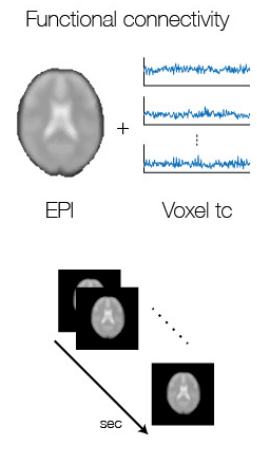

EPI timeseries

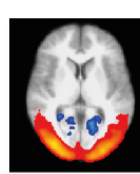

rs-MRI

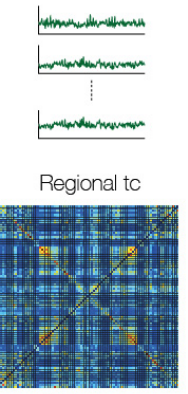

FC matrix
C

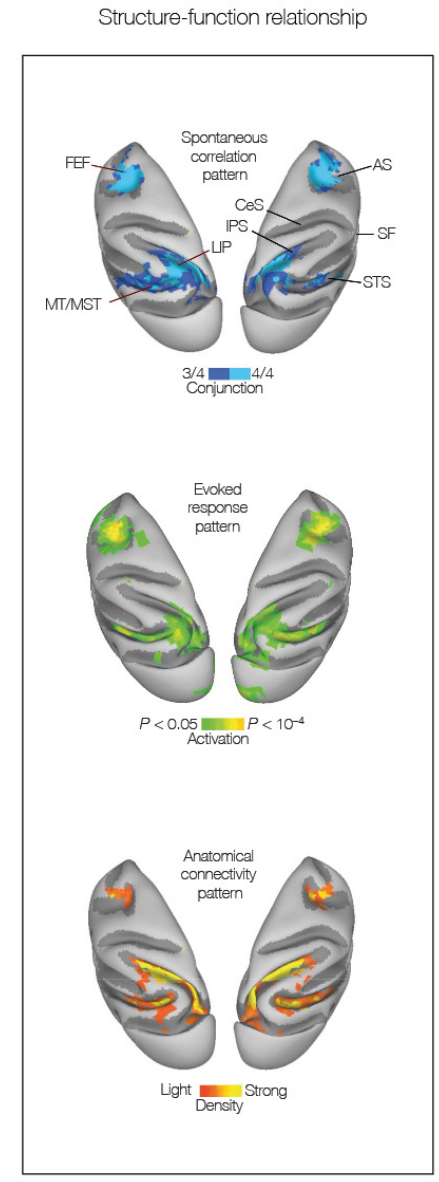

Figure 1. Methods for characterising functional and structural connectivity. Neuroimaging data can be used to extract the functional and structural connectivity in vivo. A. Typically, to obtain the topological measures of structural connectivity (SC) requires a structural MRI and diffusion tensor imaging, DTI, as well as a parcellation scheme (here we show the automatic anatomical labelling, AAL parcellation), and use tractography measures to extract the SC matrix. B. Similarly, functional connectivity (FC) can be obtained from functional neuroimaging measures of brain activity; for example, echo planar imaging (EPI) scans that sample the blood-oxygen-level dependent (BOLD) signal time course (tc) in each voxel. Combining this with a parcellation scheme can recreate the regional time courses for each of the regions in the parcellation. The FC matrix is typically created from correlating the regional time courses. C. Evidence that SC is closely linked to FC has come from neuroimaging of primates, where cortical patterns of coherent spontaneous BOLD fluctuations are similar to those of task-evoked responses and anatomical connectivity [26]. The top panel shows a conjunction map of BOLD correlations within the oculomotor system on dorsal views of the monkey atlas left and right hemisphere surfaces. The middle panel shows task-related activity in a saccadic eye movement task. The bottom panel shows the structural connectivity from the density of cells labelled by 
retrograde tracer injections into right lateral intraparietal area. Abbreviations: AS, arcuate sulcus; CeS, central sulcus; IPS, intraparietal sulcus; SF, sylvian fissure; STS, superior temporal sulcus. 
A

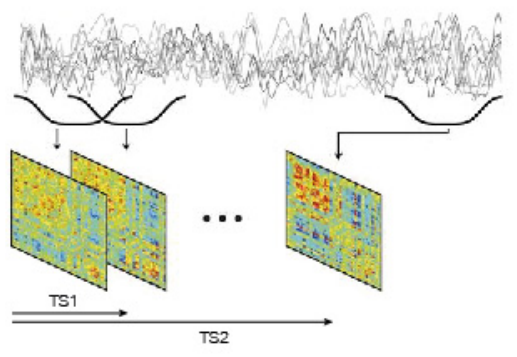

B

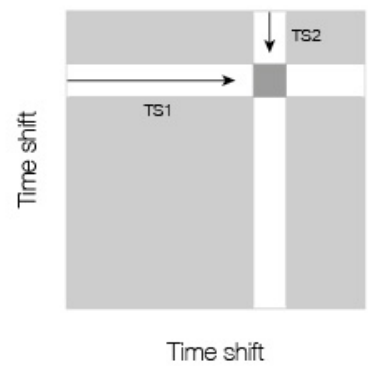

C

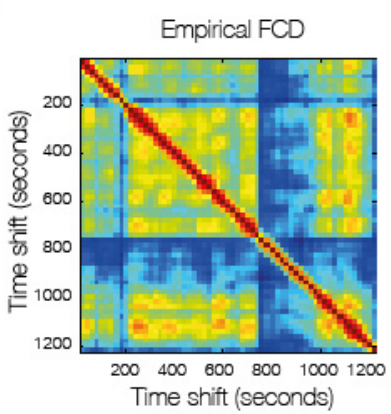

D

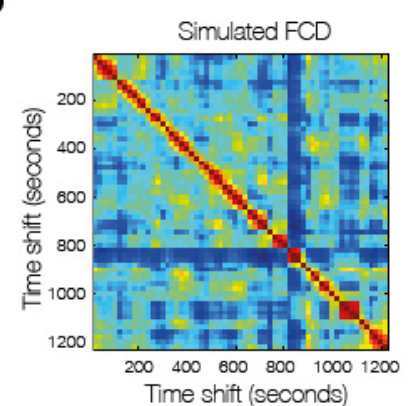

E

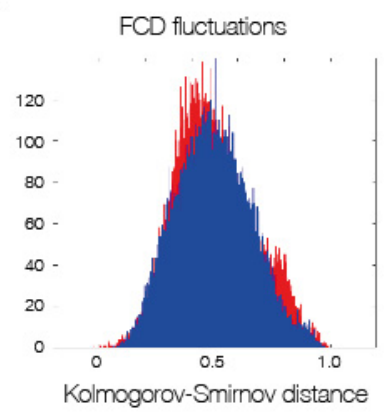

$\mathbf{F}$
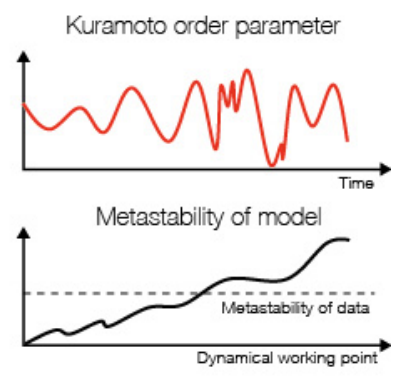

Figure 2: Methods for measuring functional connectivity dynamics (FCD). A. FCD captures the spatiotemporal organization of $\mathrm{FC}$ by representing the similarities between $\mathrm{FC}(\mathrm{t})$ matrices calculated in a short window of time (usually $60 \mathrm{sec}$ ) located at different times, e.g. TS1 and TS2. This is accomplished by splitting each full-length BOLD timeseries into a number of sliding windows, $M$. For each of these sliding window, centered at time $t$, a separate FC matrix, FC(t), can be calculated. B. The FCD is thus a symmetric matrix with dimension MxM where the (ts1, ts2) entry is defined by the Pearson correlation between the upper triangular parts of the two matrices FC(ts1) and FC(ts2). C. Thus epochs of stable FC(t) configurations are reflected around the FCD diagonal in blocks of elevated inter-FC(t) correlations. D. Simulated FCD. E. For comparing the FCD statistics, the upper triangular elements of the matrices are collected over all participants and sessions. The simulated and empirical distributions can then be compared by calculating the Kolmogorov-Smirnov distance between them, as this quantifies the maximal difference between the cumulative distribution functions of the two samples. F. The metastability can be measured as the standard deviation of the Kuramoto order parameter across time, which provides a measure the global level of synchronization of the oscillating signals. 
A

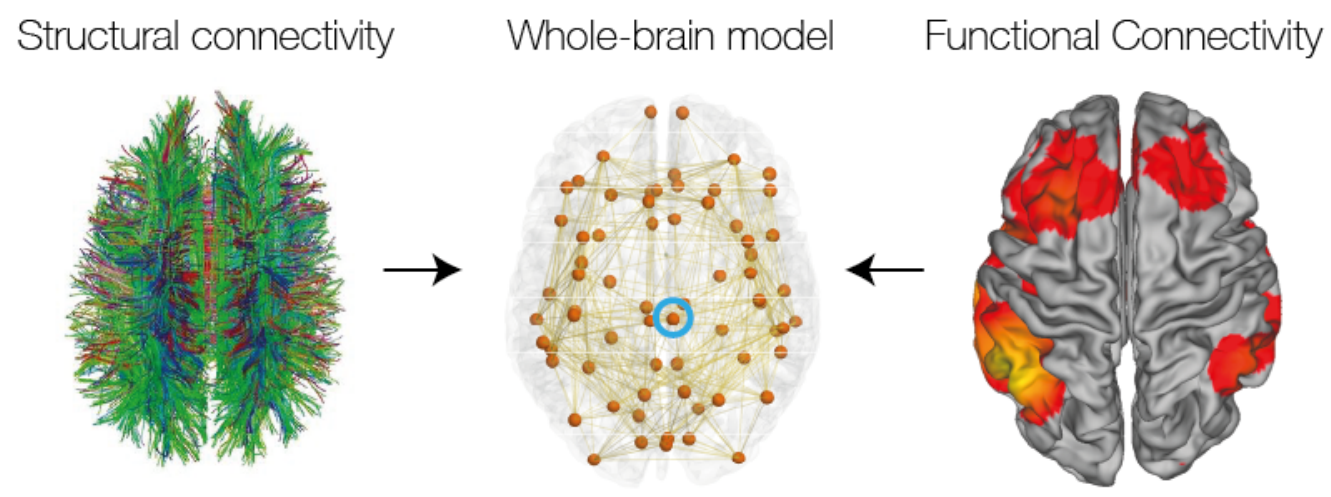

B

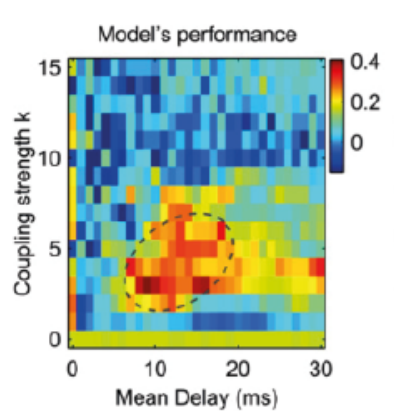

C
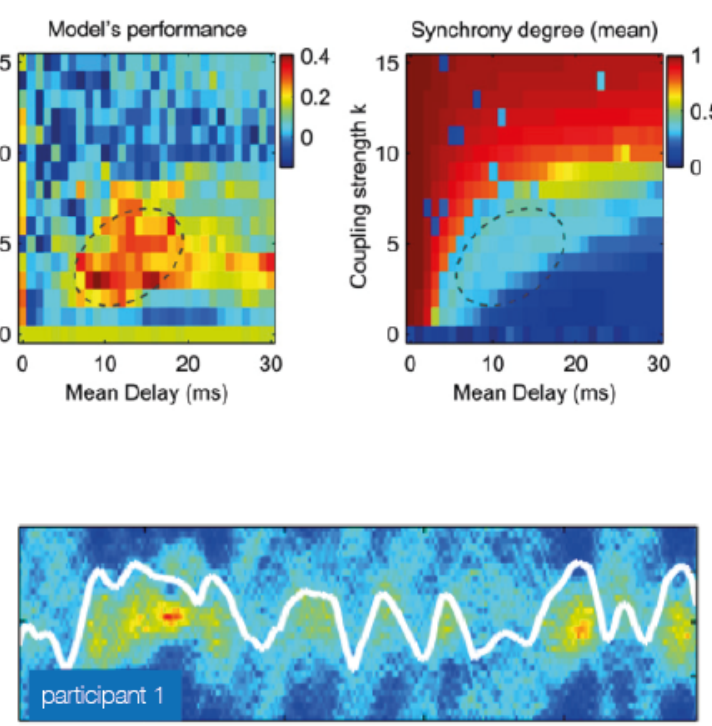
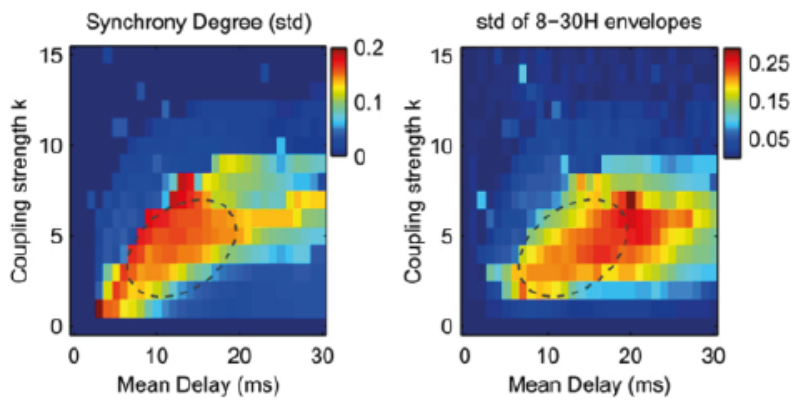

D

E

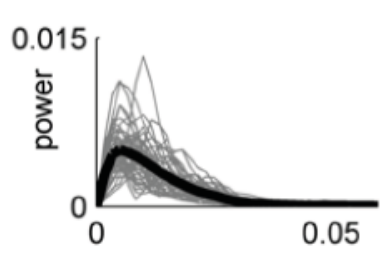

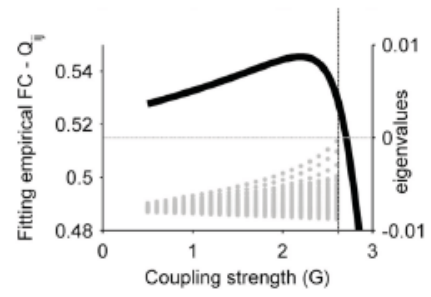

Figure 3: Meta-stability and whole-brain computational modelling.

A. Whole-brain computational models can be constructed using the structural connectivity (SC) between a given parcellation of brain regions, using either oscillatory or asynchronous local node models to fit the functional connectivity (FC) from neuroimaging data (e.g. fMRI or MEG data). B. Using an oscillatory local node model based on the Kuramoto model with only two parameters (global mean delay and coupling strength) showed maximal metastability and therefore maximal exploration of the dynamical repertoire [50]. The figures show the correlation between real and simulated FC profiles as a function of the coupling strength and the mean of the delay distribution. The area within the dashed line indicates the parameter range for which the model best estimates real MEG FC profiles. Fluctuations in the mean and standard deviation of synchrony degree are indicative of a metastable synchronization regime (two middle figures). Similarly, in the presence of time-delays, the metastable synchronization induces a modulation of the oscillators' frequency, which generates envelope fluctuations of $8-30 \mathrm{~Hz}$ oscillations. C. The temporal dynamics of phase interactions are shown for a typical participant [38]. D. The power spectrum of the Kuramoto order parameter is shown across all participants, indicative of slow processing [38]. E. Structured linear fluctuations around the spontaneous state show a characteristic critical slowing down before the bifurcation [51]. 


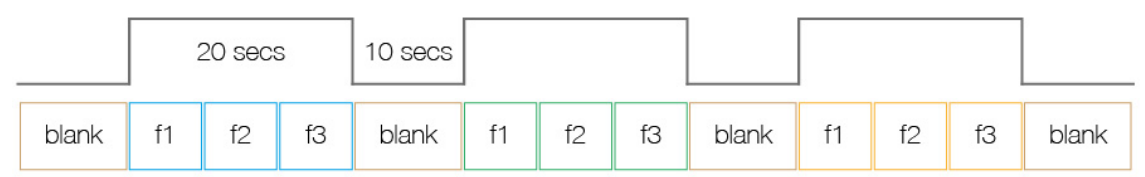

movie

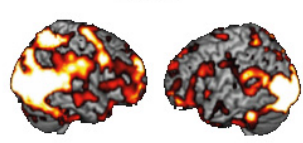

scrambled
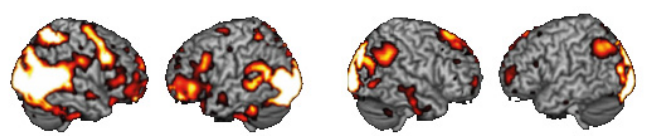

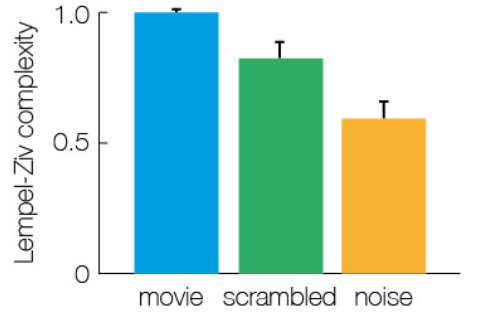

C
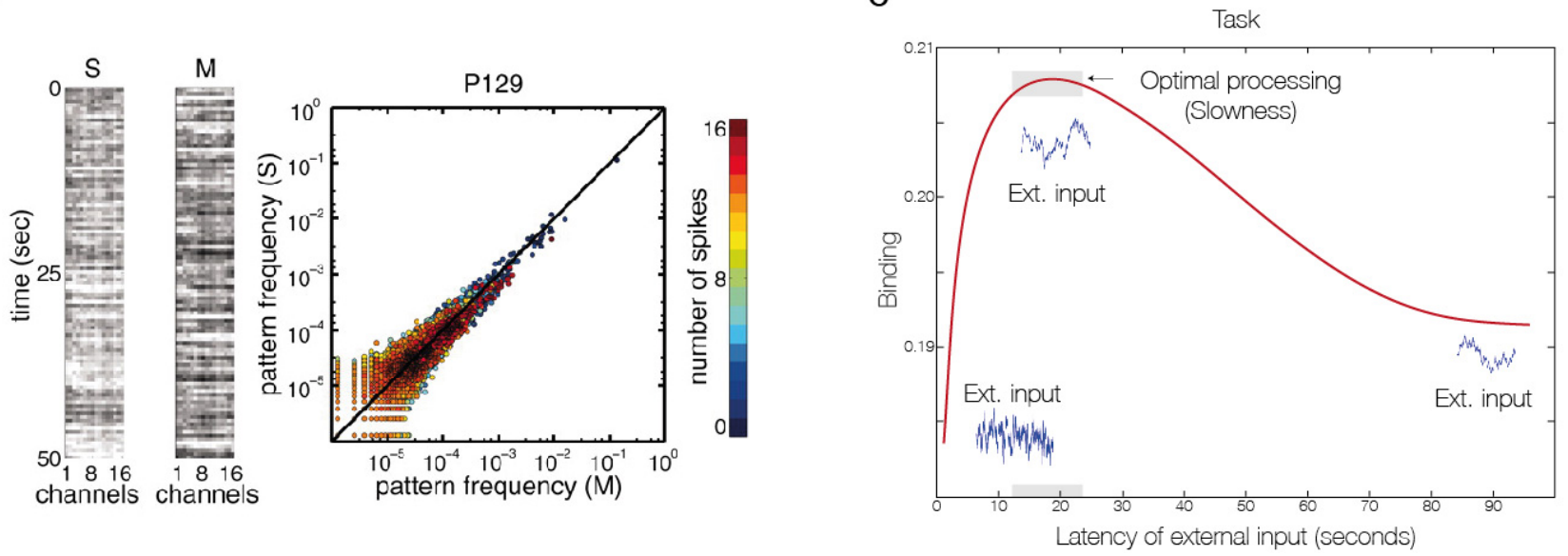

Figure 4: Slowness in processing of natural stimuli

A. The complexity of brain activity related to natural sequence of images (movie) was significantly higher than to temporally and spatially scrambled images (with preserved first-order statistics). Adapted from [63]. This suggests that the temporal binding of information in human brain is better suited to the natural, slow timing/latencies of stimuli than rapid unstructured noise. B. Similarly, recordings of neural activity in awake ferrets to natural scenes stimuli (movie, M) show that the neural activity is well matched to the spontaneous neural activity (S). C. We hypothesize that the binding (i.e. the level of encoding of information) is maximal when the brain is maximally metastable. This could be tested using a whole-brain computational model optimised to spontaneous activity but driven by external stimuli. This suggests that in order for the brain to optimally process external signals, the latencies should be on the order of the underlying latency of the timing of the resting brain, i.e. of the switching between resting brain states, which existing data suggests would be with a latency of external input of around 20 seconds. At that point, the external signal "resonates" with the underlying latency of the whole brain dynamics, leading to optimal processing. 


\section{Box 1: The Virtual Brain}

The Virtual Brain (TVB) is a freely-available public tool based on an international neuroscience project that promotes the large-scale network perspective of brain function and dysfunction, and assembles the neuroinformatics competences for the computer simulation of individual brain networks ([77]; see Figure I). This level of description allows mimicking the empirical data sets obtained from invasive and non-invasive human brain imaging. TVB provides for the first time the possibility to feed computational neuronal network models with information about brain activity (EEG, MEG), spatially highly resolved whole brain metabolic/vascular signals (fMRI) and global measures of neuronal connections (DTI) - for intact as well as pathologically altered connectivity. This enables the development of individual connectivity dependent models of oscillatory activity and to characterize the network mechanisms that underlie the genesis of brain activity. The TVB platform allows performing either simulation with systematic parameter sweeps for hypothesis testing; or fitting of parameters against a participant's brain imaging data. These approaches can lead to the development of new diagnostic and therapeutic interventions. Currently on-going projects are in the field of epilepsy, stroke and aging. Beyond the direct implications for diagnosis, treatment and prevention of network dysfunctions, the results of the Virtual Brain studies are likely to significantly contribute to the understanding of fundamental mechanisms of the brain's structure-function relationship.

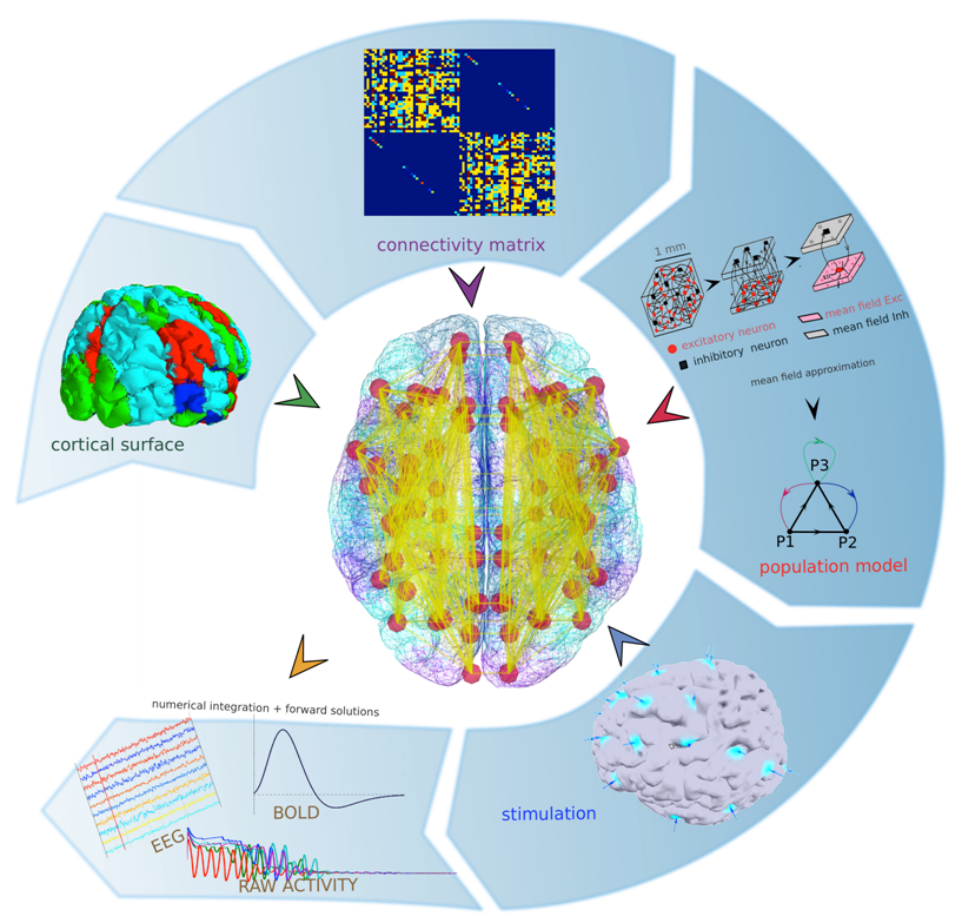

Figure I. Workflow in The Virtual Brain. A template for the cortical surface defines three-dimensional geometry and parcellation. Methods of DTI allow reconstructing connections and tract lengths of fiber bundles to define a connectivity matrix and time delays via signal transmission. Neural population models serve as network nodes generating dynamic signals due to their intrinsic dynamics and interactions with other nodes. Interventions such as lesioning of the connectivity or stimulating brain regions offer means to 
probe the brain dynamics in TVB. The resulting brain network dynamics is registered as raw Local Field Potentials (LFP), Bold fMRI, EEG and MEG signals. 


\section{Box 2: Stability and time in the human brain}

Time is of the essence in the human brain, giving rise to the time-critical neural computations allowing organisms to survive [75]. This complex brain activity during rest and cognition plays out on the background of the brain's structural connectivity, and crucially has to balance the exploration of this dynamical potential to ensure stability in the long-term [78].

Whole-brain computational models combine brain structure and activity dynamics to explore and explain the emergence of resting-state networks mechanistically. Until recently, the models have typically been either oscillatory [45-47, 49,50] or 2) asynchronous [51-53]. These models use different strategies to explain the functional connectivity of the data over time, either, in the case of oscillatory models, by maximizing the metastability of the dynamics, or, in the case of asynchronous models, by working at the border of multistability, i.e. at a dynamical point at the edge of the bifurcation where the trivial spontaneous state loses stability. In this Review, we argue that it is high time that we move away from solely describing the average of activity over time and to start to describe time-dependent activity. We also argue that the Hopf bifurcation model captures the best of both models [54, 55].

The stability of whole-brain computational models hinges on different concepts from dynamical system. The asynchronous models provide evidence that the simulated functional connectivity best matches empirically observed functional connectivity when the whole-brain network is subcritical, meaning that when there are stable attractors states, with a spontaneous state with low activity in all regions, and several excited states with high activity between selected regions. In other words, multistability around a spontaneous state defines an operating point such that system activity stochastically explores the dynamic repertoire inherent to the structural connectivity $[42,51]$.

Similarly, the oscillatory models provide evidence for the importance of metastability, which is a measure of how variable brain states are as a function of time; e.g. how the synchronization between the different brain regions fluctuates across time. These concepts of multi- and metastability for describing the dynamical systems in the brain are possible scenarios for the resting state. It remains an active area of research to determine which is a more accurate description [39]. 


\section{Outstanding Questions}

* What are the necessary and sufficient brain regions for balancing fast and slow processing?

* How does the balance between slow and fast processing change between different conscious state, e.g. between awake and different stages of sleep?

* What are the neural mechanisms by which the reward system can bias the brain to change the balance between fast and slow processing?

* What is the developmental trajectory of slowness?

* How does the balance between slow and fast processing change in specific brain disorders?

* Could slowness be a biomarker of a healthy brain? 


\section{Glossary}

\section{Attractor}

A set to which a dynamical system evolves after a long enough time. Points that get close to the attractor remain close, even under small perturbations.

\section{Bifurcation}

An abrupt qualitative change in the system's dynamics when one or more parameter pass through critical values, for instance the loss of stability and appearance of sustained oscillations.

\section{Connectome}

The complete description of the structural connections between elements of a nervous system.

\section{Criticality}

At the brink of a bifurcation, the system displays certain characteristic dynamic features, of which most are related to enhanced fluctuations.

\section{Critical slowing down}

The phenomenon that the return time of a disturbance back to equilibrium increases close to a bifurcation.

\section{Dynamical systems}

An area of applied mathematics that describes the behaviour of complex (possibly chaotic) dynamical systems as described by differential or difference equations.

\section{Diffusion tensor imaging (DTI)}

An MRI technique that takes advantage of the restricted diffusion of water through myelinated nerve fibres in the brain to infer the anatomical connectivity between brain regions.

\section{Edges}

In a brain graph, an edge between nodes (regions or neurons) indicates that the nodes are anatomically or functionally connected.

\section{Functional connectivity}

Statistical association - for example, significant correlations - between neurophysiological measurements recorded from anatomically distinct neurons or regions at several time points.

\section{Graph theory}


A branch of mathematics that deals with the formal description and analysis of graphs. A graph is defined simply as a set of nodes (vertices) linked by connections (edges), and may be directed or undirected. When describing a real-world system, a graph provides an abstract representation of the system's elements and their interactions.

\section{Hopf bifurcation}

In nonlinear dynamics, a Hopf bifurcation is a local bifurcation in which an initially stable fixed point of a dynamical system loses its stability in an oscillatory fashion.

\section{Kuramoto order parameter}

A measure of the level of synchrony between oscillators in a network.

\section{Mean-Field model}

The mean-field approximation consists of replacing the temporally averaged discharge rate of a cell with an equivalent momentary activity of a neural population (ensemble average) that corresponds to the assumption of ergodicity. According to this approximation, we characterize each cell assembly by means of its activity population rate.

\section{Metastability}

In dynamical systems refers to a state which falls outside the natural equilibrium state of the system but persists for an extended period of time.

\section{Magnetoencephalography (MEG)}

A method of measuring brain activity by detecting minute perturbations in the extracranial magnetic field that are generated by the electrical activity of neuronal populations.

\section{Multistability}

Coexistence of more than two stable steady states at given parameter values. 